\title{
Partial Dynamical Symmetry and Mixed Dynamics
}

\author{
A. Leviatan ${ }^{a}$ and N. D. Whelan ${ }^{b, c}$ \\ ${ }^{a}$ Racah Institute of Physics, The Hebrew University, Jerusalem 91904, Israel \\ ${ }^{b}$ Centre for Chaos and Turbulence Studies, Niels Bohr Institute, Copenhagen, Denmark \\ ${ }^{c}$ Division de Physique Théoriqu*, IPN, 91406 Orsay Cedex, France
}

\begin{abstract}
Partial dynamical symmetry describes a situation in which some eigenstates have a symmetry which the quantum Hamiltonian does not share. This property is shown to have a classical analogue in which some tori in phase space are associated with a symmetry which the classical Hamiltonian does not share. A local analysis in the vicinity of these special tori reveals a neighbourhood of phase space foliated by tori. This clarifies the suppression of classical chaos associated with partial dynamical symmetry. The results are used to divide the states of a mixed system into "chaotic" and "regular" classes.
\end{abstract}

PACS numbers: 05.45.+b, 03.65.Fd, 03.65.Sq, 11.30.Na

Typeset using REVTEX 
Symmetry plays a central role in affecting the character of classical and quantum dynamics. Constants of motion associated with a symmetry govern the integrability of the system. Often the symmetry in question is not obeyed uniformly, i.e. only a subset of quantum states fulfill the symmetry while other states do not. In such circumstances, referred to as partial symmetries, the symmetry of these "special" (at times solvable) eigenstates does not arise from invariance properties of the Hamiltonian. Selected examples in this category are adiabatic regular states in the stadium billiard [1]; regular quasi-Landau resonances of a hydrogen atom in strong magnetic fields [2]; discrete nuclear states embedded in a continuum of decay channels [3]; partial $S U(3)$ symmetry found in deformed nuclei [幽. Hamiltonians with partial symmetries are not completely integrable hence can exhibit stochastic behavior. As such they are relevant to the study of mixed systems with coexisting regularity and chaos [5], which are the most generic.

The effect of discrete symmetries on a mixed-phase-space system was examined in [6], while the consequences of breaking a discrete symmetry on the spectral statistics of a chaotic Hamiltonian were discussed in [7]. In the present paper we focus on continuous symmetries (associated with Lie groups) which can be conveniently studied in the framework of algebraic models. Such symmetry-based models are amenable to both quantum and classical treatments and have been used extensively in nuclear and molecular physics [8]. Their integrable limits are associated with dynamical symmetries in which the Hamiltonian is written in terms of Casimir operators of a chain of nested algebras. The eigenstates and eigenvalues are labeled by the irreducible representations (irreps) of the algebras in the chain, and are known analytically. Both the quantum and classical Hamiltonians are completely solvable [9]. Non-integrability is obtained by breaking the dynamical symmetry and may lead to chaotic dynamics [9, 10]. To address situations of mixed dynamics, an algorithm was developed [11] for constructing algebraic Hamiltonians with partial dynamical symmetries. Such Hamiltonians are not invariant under a symmetry group and yet possess a subset of "special" solvable states which do respect the symmetry. In the context of a nuclear physics model involving five quadrupole degrees of freedom, it was shown that partial dynamical 
symmetry (pds) induced a strong suppression of classical chaos [12]. This was true even though the fraction of special states vanished as $\hbar^{2}$, so one might have expected no classical effect. In order to better understand this effect, we consider a simpler model and use its pds to infer relationships between the classical and quantum dynamics of a Hamiltonian in a mixed KAM régime.

As a simple test-case we consider a model based on a $U(3)$ algebra. This algebra (with fermionic operators) was considered previously in the context of chaos, but not regarding pds [10]. Here we employ a realization in terms of three types of bosons $a^{\dagger}, b^{\dagger}, c^{\dagger}$ satisfying the usual commutation relations. The nine number-conserving bilinear products of creation and destruction operators comprise the U(3) algebra. The conservation of the total bosonnumber $\hat{N}=\hat{n}_{a}+\hat{n}_{b}+\hat{n}_{c}\left(\hat{n}_{a}=a^{\dagger} a\right.$ with eigenvalue $n_{a}$ etc. $)$ ensures that the model describes a system with only two independent degrees of freedom. All states of the model are assigned to the totally symmetric representation $[\mathrm{N}]$ of $U(3)$. One of the dynamical symmetries of the model is associated with the following chain of algebras

$$
U(3) \supset U(2) \supset U(1)
$$

Here $U(2) \equiv S U(2) \times U_{a b}(1)$ with a linear Casimir $\hat{n}_{a b}=\hat{n}_{a}+\hat{n}_{b}$ (which is also the generator of $\left.U_{a b}(1)\right)$. The generators of $S U(2)$ are $\hat{J}_{+}=b^{\dagger} a, \hat{J}_{-}=a^{\dagger} b, \hat{J}_{z}=\left(\hat{n}_{b}-\hat{n}_{a}\right) / 2$ and its Casimir $\vec{J}^{2}=\hat{n}_{a b}\left(\hat{n}_{a b}+2\right) / 4$. The subalgebra $U(1)$ in Eq. (1) is composed of the operator $\hat{J}_{z}$. A choice of Hamiltonian with a $U(2)$ dynamical symmetry is

$$
H_{0}=\omega_{a} a^{\dagger} a+\omega_{b} b^{\dagger} b=\hat{n}_{a b}-2 A \hat{J}_{z}
$$

where $\omega_{a, b}=1 \pm A$, and $A$ is introduced to break degeneracies. Diagonalization of this Hamiltonian is trivial and leads to eigenenergies $E_{n_{a}, n_{b}}=\omega_{a} n_{a}+\omega_{b} n_{b}$ and eigenstates $\left|n_{a}, n_{b}, n_{c}\right\rangle$ or equivalently $\left|N, J, J_{z}\right\rangle$ where the label $J=n_{a b} / 2$ identifies the $S U(2)$ irrep. These are states with well defined $n_{a}, n_{b}$ and $n_{c}=N-n_{a}-n_{b}$. To create a pds we add the term

$$
H_{1}=b^{\dagger}\left(b^{\dagger} a+b^{\dagger} c+a^{\dagger} b+c^{\dagger} b\right) b
$$


which preserves the total boson number but not the individual boson numbers, so it breaks the dynamical symmetry. However states of the form $\left|n_{a}, n_{b}=0, n_{c}\right\rangle$ (or equivalently $\left.\left|N, J=n_{a} / 2, J_{z}=-J\right\rangle\right)$ with $n_{a}=0,1,2, \ldots N$ are annihilated by $H_{1}$ and therefore remain eigenstates of $H_{0}+B H_{1}$. The latter Hamiltonian is not an $S U(2)$ scalar yet has a subset of $(N+1)$ "special" solvable states with $S U(2)$ symmetry, and therefore has pds. There is one special state per $S U(2)$ irrep $J=n_{a} / 2$ (the lowest weight state in each case) with energy $\omega_{a} n_{a}$ independent of the parameter B. Other eigenstates are mixed. Although $H_{0}$ and $H_{1}$ do not commute, when acting on the "special" states they satisfy

$$
\left[H_{0}, H_{1}\right]\left|n_{a}, n_{b}=0, n_{c}\right\rangle=0
$$

To break the pds we introduce a third interaction

$$
H_{2}=a^{\dagger} c+c^{\dagger} a+b^{\dagger} c+c^{\dagger} b
$$

The complete Hamiltonian is then

$$
H=H_{0}+B H_{1}+\mathrm{CH}_{2}
$$

For $B=C=0$ we have the full dynamical symmetry; for $B \neq 0, C=0$ we have partial dynamical symmetry and for $C \neq 0$ we have neither.

The classical Hamiltonian $\mathcal{H}_{c l}$ is obtained from (6) by replacing $\left(a^{\dagger}, b^{\dagger}, c^{\dagger}\right)$ by complex c-numbers $\left(\alpha^{*}, \beta^{*}, \gamma^{*}\right)$ and taking $N \rightarrow \infty$. The latter limit is obtained [9.10] by rescaling $\bar{B}=N B, \alpha \rightarrow \alpha / \sqrt{N}$ etc. and considering the classical Hamiltonian per boson $\mathcal{H}=\mathcal{H}_{c l} / N$. In the present model the latter has the form

$$
\mathcal{H}=\mathcal{H}_{0}+\bar{B} \mathcal{H}_{1}+C \mathcal{H}_{2}
$$

Number conservation imposes a constraint $\alpha^{*} \alpha+\beta^{*} \beta+\gamma^{*} \gamma=1$, so that the phase space is compact and four-dimensional with a volume $2 \pi^{2}$. The total number of quantum states is $(N+1)(N+2) / 2$. Assigning, to leading order in $N$, one state per $(2 \pi \hbar)^{2}$ volume of phase space, we identify $\hbar=1 / N$, so that the classical limit is $N \rightarrow \infty$. 
In all calculations reported below we take $A=0.8642$ and $N=60$. As a first step, we fix $\bar{B}=0.5$ and vary $C$. For the classical analysis we randomly sample the phase space and determine the fraction $\mu$ of chaotic volume. For the quantum analysis we evaluated the energy levels, calculated the nearest neighbors level spacing distribution of the unfolded spectrum and fitted it to a Brody distribution [13]. The Brody fit parameter $\omega$ is expected to be 0 for integrable systems (Poisson) and 1 for chaotic systems (GOE). As shown in Fig. 1, both of these measures indicate a suppression of chaos near $C=0$ similar to the results of Ref. [12]. To appreciate the strong effect of the pds (at $C=0$ ) on the underlying dynamics, it should be noted that the fraction of the solvable states $\left|n_{a}, n_{b}=0, n_{c}\right\rangle$ is $2 /(N+1)$, which approaches zero in the classical limit. To measure the extent to which each eigenstate $|\Psi\rangle$ has $S U(2)$ symmetry, we define variances $\sigma_{i}^{2}=\left\langle\Psi\left|\hat{n}_{i}^{2}\right| \Psi\right\rangle-\left\langle\Psi\left|\hat{n}_{i}\right| \Psi\right\rangle^{2}(i=a, b)$. A state which belongs to just one irrep of $S U(2)$ (with well defined $J, J_{z}$ ) has zero variances, while a mixed state has large variances. These variances have the same physical content as the entropies considered in Ref. [12]. It is instructive to display the average $\left\langle\hat{n}_{a}\right\rangle$ and variance of each state, as done in Fig. 2. $S U(2)$ pds is present in Fig. 2a $(B \neq 0, C=0)$, Fig. 2b is a blow up of Fig 2a and in Fig. 2c the symmetry is completely broken $(C \neq 0)$. In Figs. 2a-b we see states with zero variance. These are just the special $N+1$ states $\left(n_{b}=0\right)$ discussed before, which preserve the $S U(2)$ symmetry. In addition, we see families of states with small variance and small $\left\langle n_{b}\right\rangle$ which suggests that the presence of partial symmetry increases the purity of states other than the special ones. By contrast, in Fig. 2c we see no particular structure because of the destruction of the pds for $C \neq 0$.

Considerable insight is gained by examining the classical phase space structure in terms of action-angle variables $\alpha=\sqrt{J_{a}} \exp \left(-i \theta_{a}\right), \beta=\sqrt{J_{b}} \exp \left(-i \theta_{b}\right)$ and $\gamma=\sqrt{J_{c}}=\sqrt{1-J_{a}-J_{a}}$. The $\theta_{a}=-\pi / 2$ Poincaré section is shown in Fig. 3 for energy $E=1.0$. When $S U(2)$ pds is present $(\bar{B} \neq 0, C=0)$ we see in Figs. 3a-b a torus with $J_{b}=0$, and additional perturbed tori in its neighborhood ( $\operatorname{small} J_{b}$ ). This structure is absent when the symmetry is completely broken $(C \neq 0)$, as shown in Fig. 3c. The features in Fig 3 persist also at other energies. To understand them, we recall that for $\bar{B}=C=0$, the Hamiltonian (7) is integrable and all 
trajectories wind around invariant tori. By standard torus quantization (without turning points) the actions are quantized as $J_{i}=n_{i} \hbar=n_{i} / N(i=a, b)$. In the integrable limit quantum states are associated with toroidal manifolds in phase space. In case of a partial symmetry $(\bar{B} \neq 0, C=0)$ we are led by analogy with Eq. (4) to seek manifolds $\mathcal{M}$ in phase space on which

$$
\left.\left\{\mathcal{H}_{0}, \mathcal{H}_{1}\right\}\right|_{\mathcal{M}}=0
$$

vanishes even though the Poisson bracket is not zero everywhere. In addition, we demand $\left.\left\{\left\{\mathcal{H}_{0}, \mathcal{H}_{1}\right\}, \mathcal{H}_{0}+\mathcal{H}_{1}\right\}\right|_{\mathcal{M}}=0$ (in analogy to the quantum relation $\left[\left[H_{0}, H_{1}\right], H_{0}+H_{1}\right] \mid n_{a}, n_{b}=$ $\left.0, n_{c}\right\rangle=0$ ) so that a trajectory starting on $\mathcal{M}$ remains on $\mathcal{M}$. The solution to these conditions is the manifold $J_{b}=\beta^{*} \beta=0$, which may be interpreted as a (degenerate) torus of the $\mathcal{H}_{0}$ Hamiltonian. It is also a stable isolated periodic orbit of $\mathcal{H}_{0}+\bar{B} \mathcal{H}_{1}$. Quantization of the torus with $J_{b}=0$ proceeds exactly as before, so we correctly predict no change in the quantum energies associated with it. The manifold $\mathcal{M}\left(J_{b}=0\right)$ is the direct classical analogue of the special quantum states $\left|n_{b}=0\right\rangle$. It refers, however, to a region of phase space of measure zero, and so cannot by itself explain the observed (global) suppression of chaos. However, as suggested by Fig. 3, the presence of pds induces a quasi-regular region foliated by tori in the vicinity of the special torus. We can understand the dynamics on a finite measure of phase space by performing a perturbative calculation in the neighbourhood of $\mathcal{M}$.

For the classical perturbation calculation we set $C=0$ in Eq. (7) and treat $\bar{B}$ as an expansion parameter, assuming $\bar{B} \mathcal{H}_{1}$ in Eq. (7) is small in the neighbourhood of the special periodic orbit. We seek a generating function for the canonical transformation $(\mathbf{J}, \boldsymbol{\theta}) \rightarrow$ $(\mathbf{I}, \boldsymbol{\phi})$ such that the Hamiltonian depends only on the new actions $\mathbf{I}$ and not on the new angles $\phi$. The first-order result in standard classical perturbation theory is

$$
S(\mathbf{I}, \boldsymbol{\theta})=\mathbf{I} \cdot \boldsymbol{\theta}-2 \bar{B} I_{b}^{3 / 2}\left[\frac{I_{a}^{1 / 2} \sin \left(\theta_{a}-\theta_{b}\right)}{(\Delta \omega)}-\frac{I_{c}^{1 / 2} \sin \theta_{b}}{\omega_{b}}\right],
$$

where $\Delta \omega=\omega_{a}-\omega_{b}$ and $\mathbf{J}=\frac{\partial S}{\partial \mathbf{I}}, \boldsymbol{\phi}=\frac{\partial S}{\partial \boldsymbol{\theta}}$. There is no first order shift in the energy hence $\mathcal{H}(I)=\omega_{a} I_{a}+\omega_{b} I_{b}$ and the calculation is valid for $\bar{B} I_{b}^{3 / 2} \ll 1$. The second order correction 
reproduces well the perturbed tori on the Poincaré sections as shown in Fig. 3b. The classical variances are defined by averaging with respect to the angles $\left(\theta_{a}, \theta_{b}\right)$, e.g. $\sigma_{a, c l}^{2}=\left\langle J_{a}^{2}\right\rangle-\left\langle J_{a}\right\rangle^{2}$. To the same order in perturbation theory, $\left\langle J_{a}\right\rangle=I_{a},\left\langle J_{b}\right\rangle=I_{b}$ and the variances in $J_{a}$ and $J_{b}$ are

$$
\sigma_{a, c l}^{2}=\frac{2 \bar{B}^{2} I_{b}^{3} I_{a}}{(\Delta \omega)^{2}} \quad \sigma_{b, c l}^{2}=2 \bar{B}^{2} I_{b}^{3}\left[\frac{I_{a}}{(\Delta \omega)^{2}}+\frac{I_{c}}{\left(\omega_{b}\right)^{2}}\right] .
$$

The same calculation can also be done in quantum perturbation theory with the result

$$
\begin{aligned}
\sigma_{a}^{2} & \left.=\frac{B^{2} n_{b}}{(\Delta \omega)^{2}}\left[\left(n_{b}-1\right)^{2}\left(n_{a}+1\right)+n_{b}\left(n_{b}+1\right) n_{a}\right)\right] \\
\sigma_{b}^{2} & =B^{2} n_{b}\left[\left(\frac{n_{a}}{(\Delta \omega)^{2}}+\frac{n_{c}}{\left(\omega_{b}\right)^{2}}\right)\left(2 n_{b}^{2}-n_{b}+1\right)+\left(\frac{1}{(\Delta \omega)^{2}}+\frac{1}{\left(\omega_{b}\right)^{2}}\right)\left(n_{b}-1\right)^{2}\right] .
\end{aligned}
$$

In the semiclassical limit, $n_{a}, n_{b}, n_{c} \gg 1$, Eqs. (10) and (11) agree to leading order. In Fig. $2 \mathrm{~b}$ we show the results (denoted by $(+)$ ) of the quantum perturbation theory (to order $\left.B^{5}\right)$. We see that the first few families of states are reproduced. It is these states which we can recover from perturbation theory and whose approximate symmetry is induced by the symmetry of the special states.

The following physical picture emerges from the foregoing analysis. Near the special orbit, there are KAM tori, some of which are quantized. The quantum eigenstates lie on these tori, so knowing the classical variance of the actions of the tori tells us the variances of the states themselves, in the semiclassical limit. Large variances indicate the extent to which the corresponding states fail to respect the symmetry. This provides a measure for a separation of regular and irregular levels, as conceived by Percival [14] and which recently gained numerical support [6,15]. In the present model the quantum states can be grouped into three classes: i) the special states, which observe the symmetry; ii) the "almost special states" which are accessible by perturbation theory; iii) the rest of the states, which are mixed. As in [6], the frontier between regular states (sets (i) and (ii)) and irregular states (set (iii) ) is not sharp.

In summary, we have considered the effect of partial dynamical symmetry (pds) on the quantum and classical dynamics of a mixed system. At the quantum level, pds by definition 
implies the existence of a "special" subset of states, which observe the symmetry. The pds affects the purity of other states in the system; in particular, neighboring states, accessible by perturbation theory, possess approximately good symmetry. Analogously, at the classical level, the region of phase space near the "special" torus also has toroidal structure. As a consequence of having pds, a finite region of phase space is regular and a finite fraction of states is approximately "special". This clarifies the observed suppression of chaos. Based on these arguments and the numerical results of Ref. [12], we anticipate the suppression of chaos to persist in higher dimensional systems with partial dynamical symmetries.

This research was partly supported by the EU Human Capital and Mobility Programme, by a Canadian NSERC fellowship (N.D.W.) and by the Israel Science Foundation administered by the Israel Academy of Sciences and Humanities (A.L.). N.D.W. thanks Y. Alhassid and S. Creagh for useful discussions. 


\section{REFERENCES}

* Unité de recherche des Universités de Paris XI et Paris VI associée au CNRS.

[1] Y.Y. Bai et. al., Phys. Rev. A31, 2821 (1985).

[2] H. Friedrich and D. Wintgen, Phys. Rep. 183, 37 (1989).

[3] I. Rotter, Rep. Prog. Phys. 54, 635 (1991).

[4] A. Leviatan, Phys. Rev. Lett. 77, 818 (1996).

[5] O. Bohigas, S. Tomsovic and D. Ullmo, Phys. Rep. 223, 43 (1993).

[6] O. Bohigas, S. Tomsovic and D. Ullmo, Phys. Rev. Lett. 64, 1479 (1990).

[7] D.M. Leitner, H. Köppel and L.S. Cederbaum, Phys. Rev. Lett. 73, 2970 (1993).

[8] F. Iachello and A. Arima, The Interacting Boson Model, Cambridge Univ. Press, Cambridge (1987); F. Iachello and R.D. Levine, Algebraic Theory of Molecules Oxford Univ. Press (1995).

[9] W. Zhang, C.C. Martens, D.H. Feng and J. Yuan, Phys. Rev. Lett. 61, 2167 (1987); Y. Alhassid, A. Novoselsky and N. Whelan, Phys. Rev. Lett. 65, 2971 (1990).

[10] R.D. Williams and S.E. Koonin, Nucl. Phys. A391, 72 (1982); D. Meredith, S. Koonin and M. Zirnbauer, Phys. Rev. A37, 3499 (1988).

[11] Y. Alhassid and A. Leviatan, J. Phys. A25, L1265 (1992).

[12] N. Whelan, Y. Alhassid and A. Leviatan, Phys. Rev. Lett. 71, 2208 (1993).

[13] T.A. Brody et. al., Rev. Mod. Phys. 53, 385 (1981).

[14] I. C. Percival, J. Phys. B6, L229 (1973).

[15] B. Li and M. Robnik, J. Phys. A28, 4843 (1995). 


\section{FIGURES}

FIG. 1. Classical $(\mu)$ and quantum $(\omega)$ measures of chaos (denoted by $(\bullet)$ and $(\times)$ respectively) versus $C$ for the Hamiltonian (6) with $\bar{B}=0.5$.

FIG. 2. The values of $\left\langle n_{a}\right\rangle$ and of the variance $\sigma_{b}$ (denoted by $\diamond$ ) of each eigenstate of the Hamiltonian (6). (a) $\bar{B}=0.5, C=0$ (partial dynamical symmetry). (b) a blow up of (a) with superimposed results (denoted by $(+)$ ) of quantum perturbation theory. The families of states with low $\sigma_{b}$ have small values of $\left\langle n_{b}\right\rangle$. (c) $\bar{B}=0.3, C=0.5$ (broken symmetry).

FIG. 3. Poincaré sections $J_{b}$ versus $\theta_{b}$ at energy $E=1.0$. (a) $\bar{B}=0.5, C=0$ (partial dynamical symmetry). (b) a blow up of (a) with superimposed results (dashed curves) of classical perturbation theory. (c) $\bar{B}=0.3, C=0.5$ (broken symmetry). 


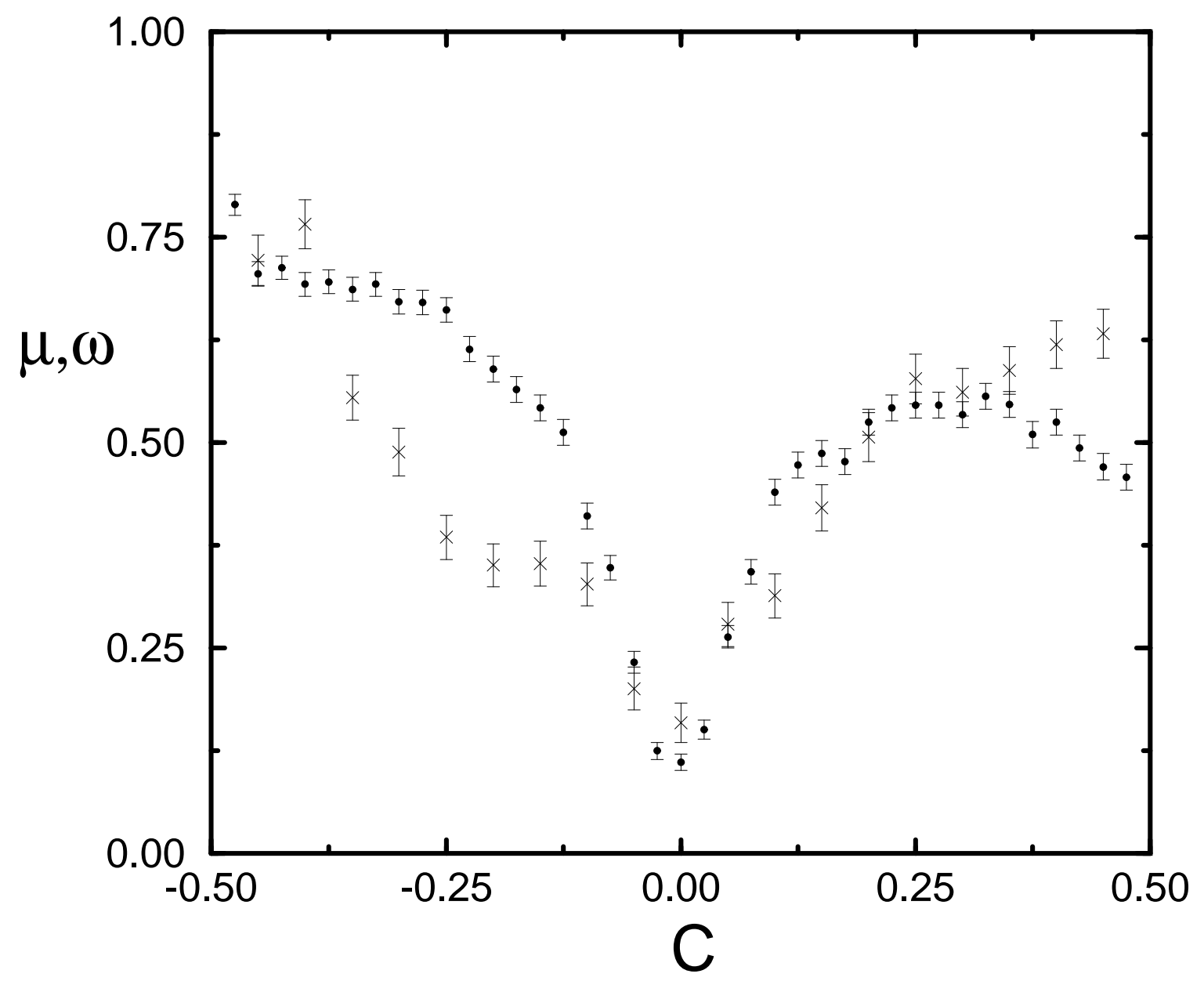




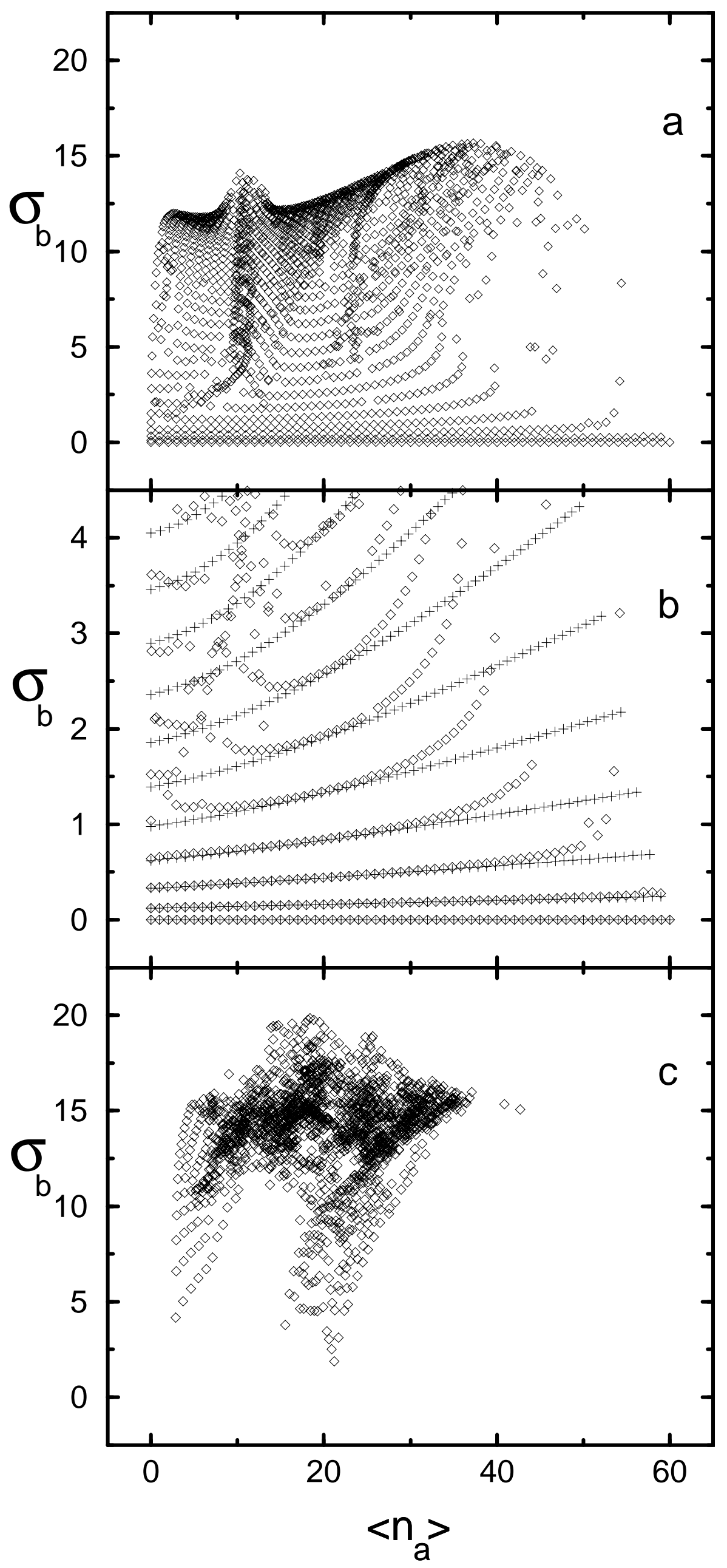




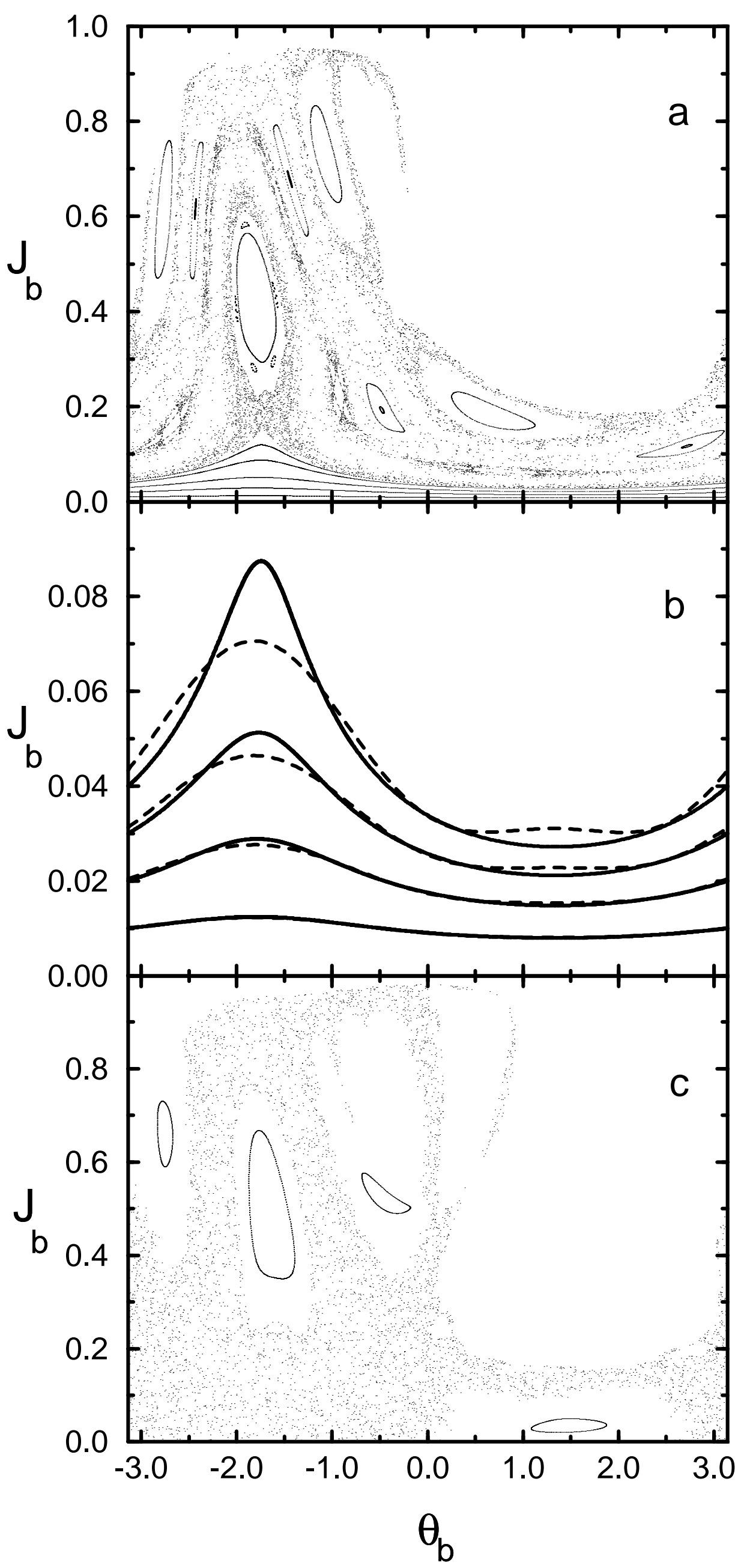

